\title{
Correlação dapresençadeespécies deflebotomíneos (Diptera: Psychodidae) com registros de casos da leishmaniose tegumentar americana no Estado de São Paulo, Brasil
}

\author{
Comelation of the presence of phlebotominae species (Diptera: Psichodidae) \\ with records of American tegumentary leishmaniasis \\ cases in the State of São Paulo, Brazil \\ Vera Lucia Fonseca de Camargo-Neves ${ }^{1}$, Almério de Castro Gomes² \\ e José Leopoldo Ferreira Antunes ${ }^{3}$
}

\begin{abstract}
Resumo $O$ estudo utilizou parâmetros ambientais associados aos coeficientes padronizados de leishmaniose tegumentar americana (LTA), no período de 1986 a 1995. Nos 140 municípios com transmissão da doença a pesquisa entomológica realizada mostrou que as espécies mais freqüentes capturadas no ambiente domiciliar, foram Lutzomyia intermedia em 87,1\% dos municípios, 53,6\% L. whitmani, 49,7\% L. migonei, 28,5\% L. pessoai e $53,6 \%$ L. fischeri. Verificou-se que as variáveis tipos de relevo e de cobertura vegetal natural influíram, significativamente $(p<0,001)$, nas médias dos coeficientes padronizados de incidência média acumulada da LTA no estado. A análise de regressão linear múltipla mostrou que a incidência da doença esteve associada, significativamente $(p=0,029)$, à presença de L. migonei nos municípios situados na região geomorfológica do Planalto Atlântico $(p=0,005)$ e, naqueles cuja cobertura vegetal predominante foi Tipo $V-$ mata $(p=0,000)$. Esta análise resgata a discussão sobre o papel vetorial de L. migonei no Estado de São Paulo.

Palavras-chaves: Lutzomyia migonei. Leishmaniose tegumentar americana. Epidemiologia.
\end{abstract}

\begin{abstract}
The study used environmental parameters associated with standardized coefficients incidence of American tegumentary leishmaniasis (ATL) in the State of Sao Paulo, Brazil, between1986 and 1995. In the 140 municipalities with leishmaniasis transmission, the entomological collection of sandflies showed the most frequent species collected in the domestic environment: Lutzomyia intermedia was present in $87.7 \%$ of the surveyed municipalities, L. whitmani in $53.6 \%$, L. migonei in $49.7 \%$, L. pessoai in $28.5 \%$ and L. fischeri in $53.6 \%$. It was found that the topographical relief and natural vegetation cover significantly influenced the mean values of standardized coefficients of accumulated incidence of ATL in the state $(p<0.001)$. Multivariable linear regression analysis showed that incidence of the disease was significantly associated with the presence of L. migonei $(p=0.029)$, in the municipalities of the Atlantic Plateau geomorphological region $(p=0.005)$ and those in which predominant vegetation cover was Type $V$ forest $(p<0.001)$. This analysis reinforces the hypothesis that L. migonei is a vector for Leishmania in São Paulo State.
\end{abstract}

Key-words: Lutzomyia migonei. American cutaneous leishmaniasis. Epidemiology.

Com a devastação da cobertura florestal primitiva no Estado de São Paulo verificou-se a redução na notificação de leishmaniose tegumentar americana (LTA) a casos esporádicos. A partir da década de 80, no entanto, novos casos da doença foram notificados, cuja transmissão foi identificada como devida a fatores antrópicos, sem vínculos com grandes ecossistemas florestados. Nessa nova feição da doença, marco de uma transição do seu perfil epidemiológico, a distribuição espacial da autoctonia dos casos ampliou-se, com cerca de $25 \%$ dos municípios do Estado apresentando ao menos a notificação de um único caso ${ }^{414}$.

A re-emergência da LTA sob novo perfil epidemiológico não apresentou risco diferenciado entre os grupos de idade e sexo e sua transmissão parece não poder ser atribuída a uma única espécie de

1. Superintendência de Controle de Endemias/SUCEN, São Paulo, SP, 2. Departamento de Epidemiologia da Faculdade de Saúde Pública da Universidade de São Paulo, SP. 3. Departamento de Odontologia Social da Faculdade de Odontologia da Universidade de São Paulo, SP. Endereço para correspondência: Drª Vera Lucia Fonseca de Camargo-Neves. R. Paula Souza 166/1ำ andar, 01027-000 São Paulo, SP Tel: $11227-0622$ r: 214, Fax: 11 227-0622 r: 229.

e-mail: vera@sucen.sp.gov.br.

Recebido para publicação em 12/2/2001 
flebotomíneo ${ }^{11}{ }^{13}$. No entanto, atribui-se preponderante papel vetorial da Leishmania (Viannia) braziliensis à Lutzomyia intermedia (Lutz \& Neiva) no ambiente domiciliar, e à L. whitmani (Antunes \& Coutinho) e Lutzomyia migonei (França) no ambiente extradomiciliar ${ }^{14} 8$.

No estado atual de complexidade da transmissão da LTA no sudeste do Brasil, sobremaneira, pela elevada alteração ambiental, evidencia-se a necessidade de novos estudos voltados à relação entre a presença de vetores, condições ambientais e a incidência da doença, sabendo-se que a distribuição geográfica da LTA é influenciada por fatores climáticos, topográficos e pela vegetação predominante que irão determinar a distribuição dos flebotomíneos ${ }^{4}{ }^{14}{ }^{15}$. Assim sendo, o presente estudo procurou correlacionar a presença de cinco espécies de flebotomíneos identificados como suspeitos de veicularem a LTA com diferentes condições ambientais e a notificação de casos da doença no Estado de São Paulo. Além disso, procurou-se evidenciar a contribuição de $L$. migonei face as novas observações feitas durante a investigação.

\section{MATERIAL E MÉTODOS}

Para a caracterização da presença e distribuição das populações flebotomíneas nos focos da doença, foram utilizados os dados de pesquisas entomológicas realizadas, no período de 1986 a 1995, pela Superintendência de Controle de Endemias (SUCEN), da Secretaria de Estado de Saúde de São Paulo. As capturas de flebotomíneos eram desencadeadas quando da realização de atividades de investigação de foco da LTA. Para tanto, lançava-se mão de armadilhas elétricas - tipo CDC, armadilha de Shannon e capturas manuais. Os locais corresponderam ao ambiente domiciliar, peri e intradomicílio, e à margem da mata, sempre próximas às residências. Seguindo as normas do Programa de Controle da LTA $^{19}$, as pesquisas entomológicas tiveram caráter qualitativo, realizadas nas primeiras três horas da noite, para as pesquisas com armadilha de Shannon e manuais, cujo ritmo das capturas obedeceram ao conhecimento e a origem dos casos suspeitos, e realizadas por período de 12 horas quando utilizadas as armadilhas luminosas.

As taxas de incidência médias foram calculadas a partir dos casos autóctones notificados ao Centro de Vigilância Epidemiológica (CVE) da Secretaria de Estado da Saúde, SP, com data de início de manifestações clínicas entre 1986 a 1995, e a padronização por sexo e faixa etária dos coeficientes de incidência média acumulada foi efetuada segundo os procedimentos descritos por Daniel ${ }^{6}$.
Considerando o estado de transição epidemiológica LTA, a análise ecológica procurou focalizar os espaços geográficos da transmissão com base na divisão geomorfológica do Estado, onde cada município foi considerado como unidade geográfica e categorizado segundo as 5 divisões geomorfológicas ${ }^{16}$ : Planalto Ocidental; Cuesta Basálticas; Depressão Periférica; Planalto Atlântico e Província Costeira. Também, foram utilizados dados da cobertura vegetal natural, obtidos do Inventário Florestal do Estado de São Paulo ${ }^{17}$; onde as 11 categorias de cobertura foram re-classificadas, para cada município, em 7 tipos de agrupamentos: I - cerrado e/ou resíduo de mata; II - capoeira e/ou pequena área de reflorestamento; III - cerradão, cerrado e/ou pequena área de reflorestamento; IV - grande área de reflorestamento; V - mata; VI - resíduo de mata e VII capoeira e/ou resíduo de mata.

A análise estatística foi efetuada através do programa SPSS 8.0, 1997. Uma vez constituído o banco de dados, procedeu-se a análise de regressão linear simples entre o coeficiente padronizado de incidência de LTA em cada município e os valores correspondentes relativos à coleta de flebotomíneos e às informações ambientais e climáticas. $O$ modelo stepwise da análise de regressão linear múltipla permitiu selecionar aqueles indicadores que mais se associaram às variações observadas na distribuição geográfica da LTA no Estado de São Paulo ${ }^{12}$.

\section{RESULTADOS}

Entre 1986 e 1995, o CVE registrou 3.380 casos de LTA distribuídos pelo estado. Os coeficientes padronizados, segundo sexo e faixa etária, de incidência média acumulada variaram de 0,00 a $3.445,85$ casos/ 100.000hab, sendo que o maior número de municípios com os maiores coeficientes padronizados de incidência média acumulada, estão situados, principalmente, a leste do estado (Figura 1).

No período, foram realizadas 1.513 pesquisas entomológicas pela SUCEN em 159 municípios, representando $61,6 \%$ daqueles com registro de caso autóctone da doença. Destes, em 151 (95\%) foram constatadas a presença de flebotomíneos. Nestes municípios $L$. intermedia foi a espécie encontrada em
$88,1 \%$, seguido de L. fischeri com $53,6 \%$; L. whitmani com 53,6\%; L. migonei com $49,7 \%$ e L. pessoai com 28,5\% (Tabela 1 e Figura 2).

A distribuição dos municípios com transmissão de LTA e pesquisa para flebotomíneos referem-se a 140 (54,3\%). Desses em $136(52,7 \%)$ a pesquisa foi positiva, tendo sido detectada pelo menos uma das cinco espécies mais freqüentes. Em 122 (87,1\%) municípios onde ocorreu a transmissão de LTA foi verificada a presença de $L$. intermedia, sendo, portanto, a espécie mais freqüente e distribuída por todas as regiões do Estado.

Analisando-se a relação entre a presença de uma das cinco espécies de flebotomíneo e a média dos coeficientes padronizados de incidência de LTA, 


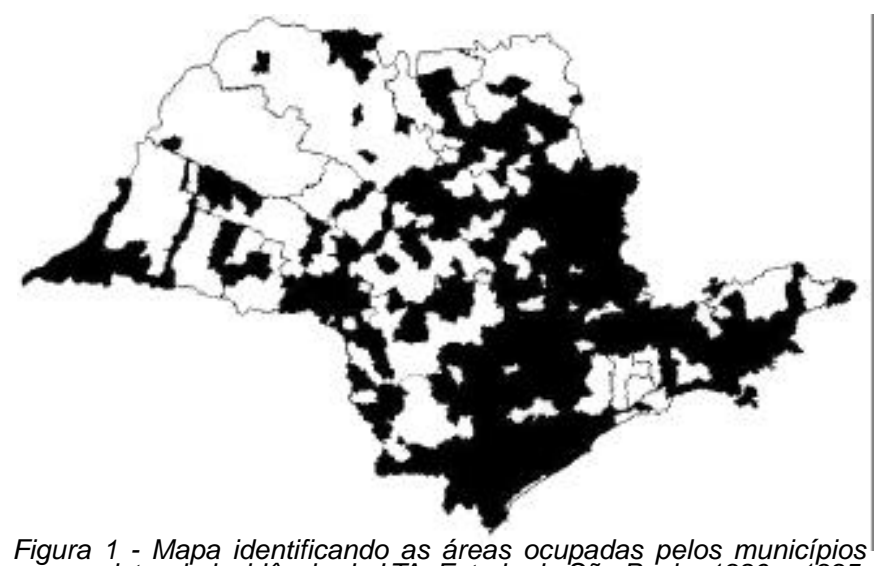

Figura 1 - Mapa identificando as áreas ocupadas pelos municípios
com registro de incidência de LTA, Estado de São Paulo, 1986 a 1995.

Tabela 1 - Distribuição proporcional da presença e ausência de flebotomíneos em 151 municípios com pesquisa entomológica. Estado de São Paulo, 1986 a 1995.

\begin{tabular}{|c|c|c|c|c|c|}
\hline \multirow[b]{2}{*}{ Espécie* } & \multicolumn{2}{|c|}{ Município } & \multirow[b]{2}{*}{ Espécie* } & \multicolumn{2}{|c|}{ Município } \\
\hline & $\mathrm{n}^{\circ}$ & $\%$ & & $\mathrm{n}^{\circ}$ & $\%$ \\
\hline Lutzomyia intermedia & 133 & 88,1 & Lutzomyia pascalei & 7 & 4,6 \\
\hline Lutzomyia fischeri & 81 & 53,6 & Lutzomyia lenti & 7 & 4,6 \\
\hline Lutzomyia whitmani & 81 & 53,6 & Lutzomyia cortelezzii & 7 & 4,6 \\
\hline Lutzomyia migonei & 75 & 49,7 & Lutzomyia arthuri & 6 & 4,0 \\
\hline Lutzomyia pessoai & 43 & 28,5 & Lutzomyia ayrozai & 6 & 4,0 \\
\hline Lutzomyia firmatoi & 31 & 20,5 & Lutzomyia pestanai & 5 & 3,3 \\
\hline Lutzomyia monticola & 29 & 19,2 & Lutzomyia longipalpis & 4 & 2,6 \\
\hline Lutzomyia alphabetica & 17 & 11,3 & Lutzomyia edwardsi & 3 & 2,0 \\
\hline Lutzomyia shannoni & 12 & 7,9 & Lutzomyia borgmeieri & 3 & 2,0 \\
\hline Lutzomyia Iloydi & 11 & 7,3 & Lutzomyia petropolitana & 2 & 1,3 \\
\hline Lutzomyia misionensis & 9 & 6,0 & Lutzomyia lanei & 1 & 0,7 \\
\hline
\end{tabular}

${ }^{*}$ Classificação segundo Young \& Duncan, $1994^{20}$

FONTE: SUCEN - SES

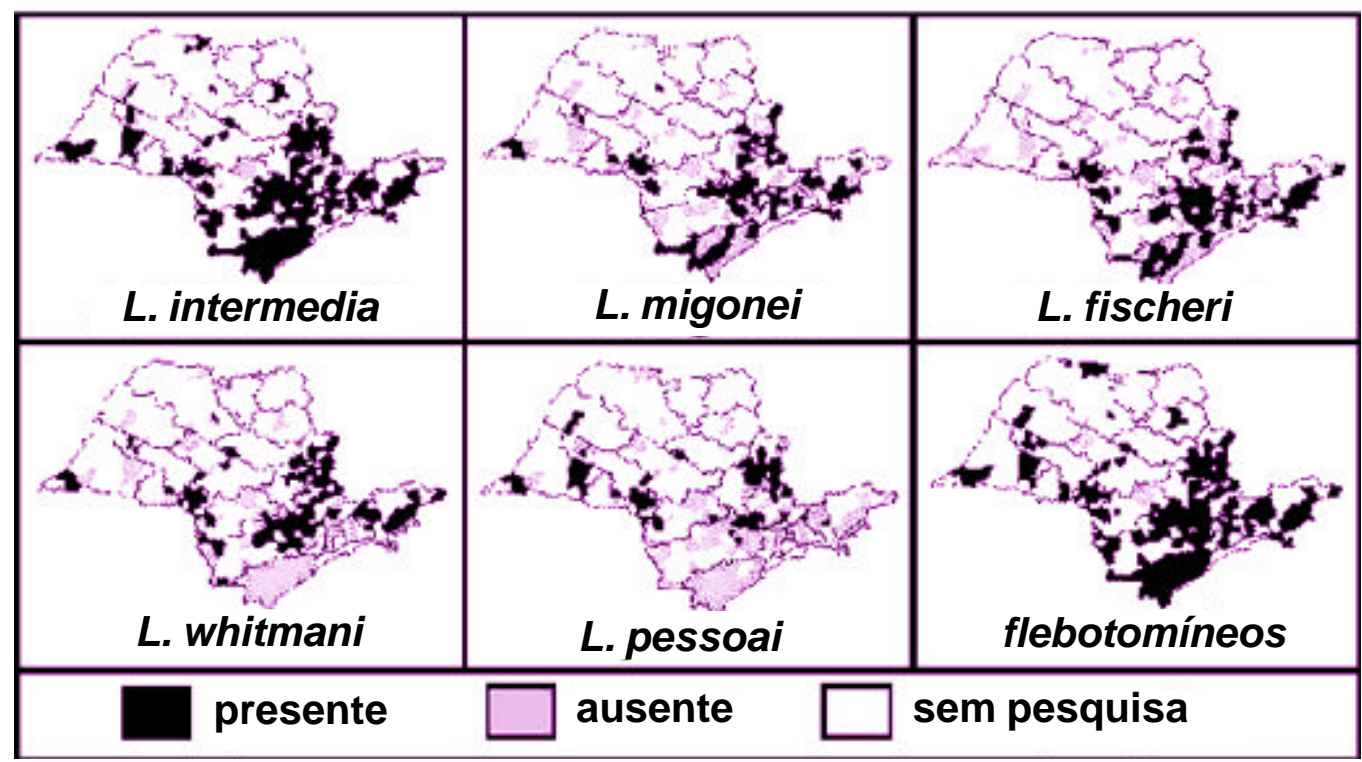

Figura 2 - Mapas identificando as áreas ocupadas pelos municípios com pesquisa de flebotomíneos, Estado de São Paulo, 1986 a 1995. 
verificou-se que a espécie de flebotomíneo não influiu significativamente $(p=0,475)$ na média do coeficiente padronizado de incidência de LTA no Estado de São Paulo, nos 136 municípios com a presença de pelo menos uma das cinco espécies mais freqüentes de flebotomíneo (Tabela 2). A análise comparativa entre os municípios entre os quais foi detectada a presença

Tabela 2 - Relação entre a detecção da presença de Lutzomyia intermedia, Lutzomyia whitmani, Lutzomyia migonei, Lutzomyia pessoai e/ou Lutzomyia fischeri sobre a média dos coeficientes padronizados de incidência de Leishmaniose Tegumentar Americana. Estado de São Paulo, 1986 a 1995.

\begin{tabular}{lrrr}
\hline & \multicolumn{2}{c}{ Municípios } & Coeficiente* \\
\cline { 2 - 3 } Espécie & $\mathrm{n}^{\circ}$ & $\%$ & Incidência \\
\hline L. intermedia & 122 & 89,7 & 180,3 \\
L. whitmani & 78 & 57,4 & 168,3 \\
L. migonei & 70 & 51,5 & 239,3 \\
L. pessoai & 41 & 30,2 & 94,5 \\
L. fischeri & 76 & 55,9 & 199,2 \\
\hline Total & & & \\
\multicolumn{1}{c}{ A } & 136 & 100,0 & 169,8 \\
B & 4 & - & 3,5 \\
C & 118 & - & 23,7 \\
\hline
\end{tabular}

*coeficiente padronizado, segundo sexo e faixa etária, de incidência média acumulada por 100.000 habitantes.

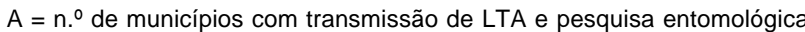
positiva.

$\mathrm{B}=\mathrm{n} .^{\circ}$ de municípios com transmissão de LTA e pesquisa entomológica negativa.

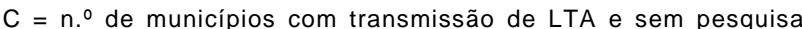
entomológica.

de pelo menos uma das cinco espécies de flebotomíneos e aqueles em que a pesquisa entomológica foi negativa, apresentou diferença significativa $(p=0,026)$ em relação a média dos coeficientes padronizados de incidência. Além disso, pode-se observar diferença significativa quanto a média dos coeficientes padronizados de incidência de LTA entre os municípios em que foram realizadas as pesquisas de flebotomíneos e aqueles em que a pesquisa não foi realizada $(p<0,001)$.

Por outro lado, a análise comparativa entre as cinco espécies de flebotomíneos, mostrou que as duas únicas diferenças significativas encontradas, ao nível de significância de $p<0,1$, indicaram que $L$. pessoai foi a espécie que esteve associada a um nível de incidência de LTA significativamente menos elevado que L. migonei $(p=0,073)$ e $L$. fischeri $(p=0,055)$.

Quando analisados os municípios com transmissão de LTA e presença de uma ou mais espécies de flebotomíneos, por região geomorfológica, verificou-se que o maior número de municípios estiveram concentrados em duas regiões topográficas, o Planalto Atlântico e a Depressão Periférica (Tabela 3), sendo a espécie $L$. intermedia a mais freqüente em todas as regiões topográficas do Estado; destaca-se ainda as ausências de $L$. whitmani e $L$. pessoai na região da Província Costeira.
Tabela 3 - Distribuição proporcional dos municípios com transmissão de Leishmaniose Tegumentar Americana e a média dos coeficientes padronizados de incidência média acumulada por 100.000 habitantes, segundo a região topográfica. Estado de São Paulo, 1986 a 1995.

\begin{tabular}{lrrc}
\hline & \multicolumn{2}{c}{ Municípios } & Coeficiente \\
\cline { 2 - 3 } Região topográfica & n.o & $\%$ & Incidência* \\
\hline Planalto ocidental & 54 & 20,9 & 43,7 \\
Cuestas basálticas & 43 & 16,7 & 30,8 \\
Depressão periférica & 65 & 25,2 & 68,9 \\
Planalto atlântico & 77 & 29,8 & 149,7 \\
Província costeira & 19 & 7,4 & 326,8 \\
\hline Total & 258 & 100,0 & 100,4 \\
\hline
\end{tabular}

* coeficiente padronizado, segundo sexo e faixa etária, de incidência média acumulada por 100.000 habitantes.

Em relação à cobertura vegetal natural, 195 (75,6\%) dos municípios com transmissão apresentaram cobertura bastante modificada, com tipos fisionômicos de porte arbóreo menores como cerrado e capoeira ou estavam em áreas de reflorestamento (Tipos I a IV). A análise comparativa dos tipos de cobertura vegetal natural em relação aos coeficientes padronizados de incidência, mostrou que o tipo de vegetação influiu significativamente na média do coeficiente padronizado de incidência de LTA, ao nível de significância $p<0,001$, embora não se tenha verificado diferença significativa entre as médias dos coeficientes padronizados de incidência para cada tipo de vegetação em relação a média do Estado (Tabela 4).

Tabela 4 - Distribuição proporcional dos municípios com transmissão de Leishmaniose Tegumentar Americana e média dos coeficientes padronizados de incidência media acumulada por 100.000 habitantes, segundo o tipo de cobertura vegetal natural. Estado de São Paulo, 1986 a 1995.

\begin{tabular}{|c|c|c|c|}
\hline \multirow[b]{2}{*}{ Tipo de Cobertura - Vegetal Natural } & \multicolumn{2}{|c|}{ Municípios } & \multirow{2}{*}{$\begin{array}{r}\text { Coeficiente } \\
\text { incidência }\end{array}$} \\
\hline & $\mathrm{n}^{0}$ & $\%$ & \\
\hline Tipo I - cerrado e/ou resíduo de mata & 18 & 7,0 & 62,8 \\
\hline $\begin{array}{l}\text { Tipo II - capoeira e/ou pequena } \\
\text { área de reflorestamento }\end{array}$ & 60 & 23,3 & 62,7 \\
\hline $\begin{array}{l}\text { Tipo III - cerradão, cerrado e/ou pequena } \\
\text { área de reflorestamento }\end{array}$ & 42 & 16,3 & 33,7 \\
\hline Tipo IV - grande área de reflorestamento & 75 & 29,1 & 45,4 \\
\hline Tipo V - mata & 29 & 11,2 & 05,7 \\
\hline Tipo VI - resíduo de mata & 4 & 1,6 & 99,8 \\
\hline Tipo VII - capoeira e/ou resíduo de mata & 30 & 11,6 & 34,1 \\
\hline Total & 258 & 100,0 & 00,4 \\
\hline
\end{tabular}

* coeficiente padronizado, segundo sexo e faixa etária, de incidência média acumulada por 100.000 habitantes.

Considerando-se a distribuição dos municípios com transmissão de LTA para cada tipo de cobertura vegetal natural e as cinco espécies de flebotomíneos mais freqüentes, verificou-se que a espécie predominante em todos tipos de cobertura vegetal natural foi a L. intermedia (Figuras 2 e 3). Pode-se observar, também, a ausência de $L$. fischeri em municípios classificados no Tipo VI (resíduo de mata), estes municípios estão situados na região geomorfológica do Planalto 
Ocidental, região que apresentou o menor número de municípios com a presença desta espécie (Figuras 1, 2 e 3).

Quando realizada análise de regressão linear múltipla (Tabela 5), para a definição das áreas de maior risco de transmissão de LTA, verificou-se que as variáveis selecionadas para o modelo foram: o tipo de relevo - Planalto Atlântico, o tipo de cobertura vegetal natural Tipo V - mata e a presença de $L$. migonei. Neste modelo considerou-se, também, a variável isoterma média anual (dados não mostrados), uma vez que o aumento de temperatura contribuiu para explicar o aumento dos indicadores de LTA, quando controlados outros fatores. As Figuras 1 a 3 mostram as áreas ocupadas pelos municípios com transmissão de LTA, bem como aquelas com condições de vegetação, relevo

\begin{tabular}{|c|c|c|c|c|c|}
\hline \multirow{2}{*}{$\begin{array}{l}\text { Parâmetros de } \\
\text { regressão }\end{array}$} & \multicolumn{3}{|c|}{ Vegetação } & \multirow{2}{*}{$\begin{array}{c}\text { Aumento de temperatura } \\
\text { (5 faixas) }\end{array}$} & \multirow{2}{*}{$\begin{array}{c}\text { Relevo - } \\
\text { Planalto Atlântico }\end{array}$} \\
\hline & Constante & Tipo V - mata & L. migonei & & \\
\hline Coeficiente & $-511,692$ & 306,343 & 130,601 & 152,945 & 224,878 \\
\hline Coeficiente padronizado & - & 0,300 & 0,169 & 0,336 & 0,280 \\
\hline Erro padrão & 162,989 & 79,846 & 59,136 & 44,950 & 78,001 \\
\hline $\mathrm{t}$ & $-3,139$ & 3,837 & 2,208 & 3,403 & 2,883 \\
\hline$p$ & 0,002 & 0,000 & 0,029 & 0,001 & 0,005 \\
\hline $\mathrm{R} 2$ & 0,240 & & & & \\
\hline R2 ajustado & 0,217 & & & & \\
\hline Erro padrão & 344,056 & & & & \\
\hline $\mathrm{F}$ & 10,496 & & & & \\
\hline
\end{tabular}

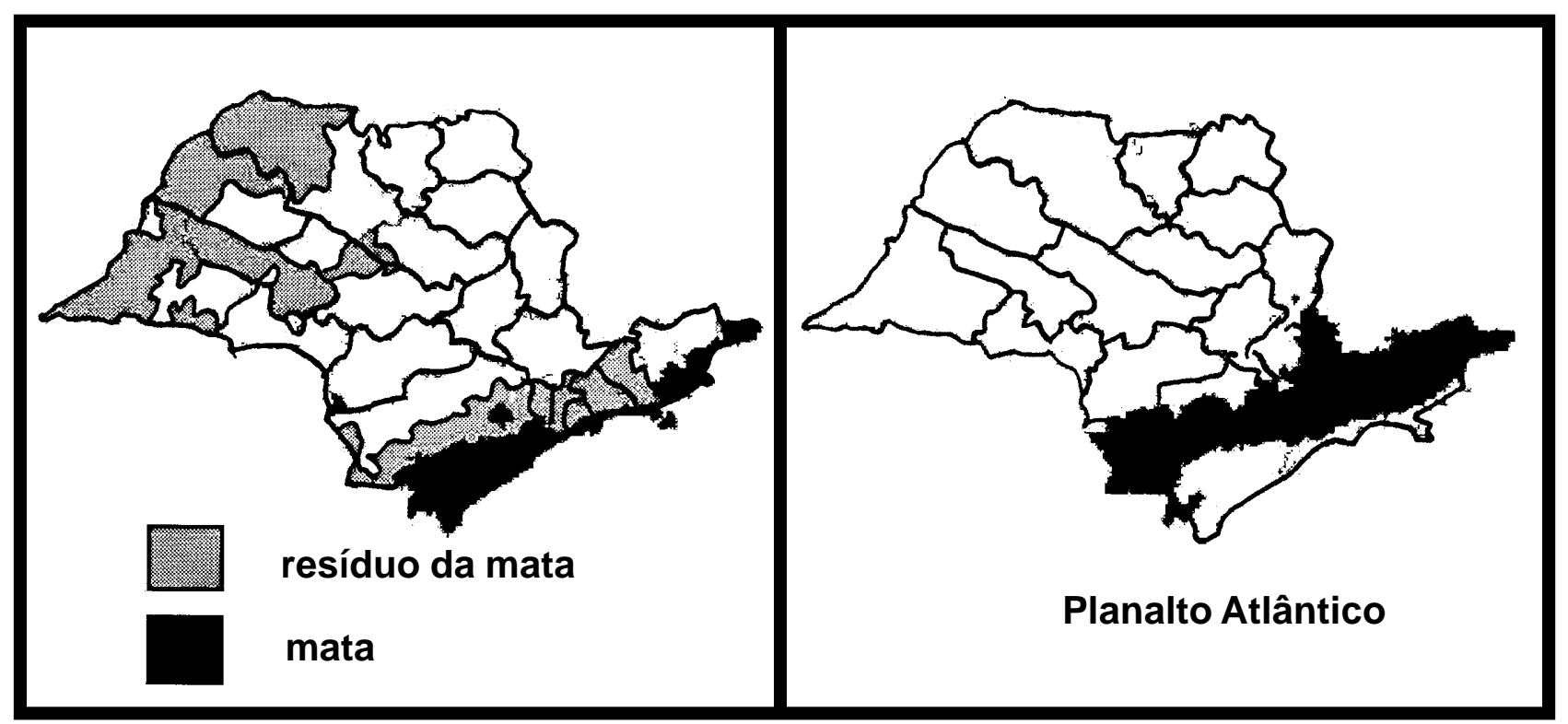

Figura 3 - Mapas identificando as áreas ocupadas pelos municípios com cobertura vegetal de mata e resíduo de mata e região topográfica do Planalto Atlântico, Estado de São Paulo.

e vetores identificados pelo modelo de análise linear múltipla, permitindo a visualização de sua superposição.

Considerou-se, também, a hipótese de haver ocorrido interação entre a presença de mais de um tipo de vetor no mesmo município, mas esta análise não melhorou a eficácia explicativa do modelo. Assim, pode-se supor que a simples detecção de uma das espécies de flebotomíneos de maior importância epidemiológica, já configura fator de risco para a LTA, não sendo este risco potencializado pela detecção de mais de uma espécie. Cabe ressaltar que este registro refere-se apenas aos tipos de vetores $\mathrm{e}$ não a densidade deles, o que não foi medido.

\section{DISCUSSÃO}

A eficácia de um programa de controle de vetores está na escolha dos indicadores mais sensíveis e no monitoramento subseqüente. A análise de informações oriundas do programa sempre contribuirá para elevação 
de seu status. A LTA apresenta dificuldades na mensuração dos indicadores entomológicos, sobretudo porque o comportamento dos vetores está influenciado por fatores ambientais submetidos à atividade antrópica. Em especial modo, a adequação das atividades do programa exige permanente atualização da modalidade de transmissão. No Estado de São Paulo, tal modalidade sempre foi complexa por conta da distribuição simpátrica das espécies antropofílicas e supostos ciclos efêmeros da Leishmania no ambiente extraflorestal. Ainda que possa ser evidente o hábito silvestre de L. whitmani e L. pessoai, apenas o dado qualitativo registrado no órgão de controle, não permitiu estratificar suas importâncias no ambiente antrópico. Mesmo assim, foi possível discutir a distribuição das cinco espécies de importância sob diferentes planos geográficos e sua associação com a incidência da doença.

As cinco espécies em questão têm se envolvido em diferentes situações de transmissão. Pessôa \& Barretto ${ }^{18}$ e Forattini ${ }^{9}$ demonstraram, no Estado de São Paulo, que L. whitmanie L. migonei poderiam ser incriminadas como espécies vetoras. Em município cearense, situado na Serra de Baturité, Azevedo e Rangel ${ }^{1}$ relataram a possibilidade de ambas espécies infectadas com Leishmania poderem invadir os habitats peridomésticos. Semelhante quadro foi verificado em Afonso Cláudio, município de Espírito Santo, situado a $650 \mathrm{~m}$ do nível do mar, onde L. migonei, L. whitmanie $L$. intermedia, foram as espécies vetoras da L. braziliensis no ambiente domiciliar, sendo que a primeira e a segunda espécies estariam fazendo a ponte entre o ambiente silvestre e domiciliar ${ }^{7}$.

A análise da distribuição das cinco espécies de flebotomíneos e da distribuição dos municípios com transmissão de LTA, segundo as médias dos coeficientes padronizados de incidência (Figuras 1 e 2), mostrou que houve sobreposição dos municípios que apresentaram maiores coeficientes padronizados de incidência e a presença de L. migonei, L. fischeri e L. intermedia (Tabela 2). Estes municípios estão situados na região geomorfológica do Planalto Atlântico e da Província Costeira e apresentaram como tipos de cobertura vegetal natural Tipo V - mata, Tipo IV grandes áreas de reflorestamento e Tipo VII - capoeira e resíduo de mata, mostrando, com isso, a influência da cobertura vegetal e do relevo na distribuição dos vetores. Gomes e $\operatorname{cols}^{15}$, analisando a distribuição geográfica dos flebotomíneos de importância na distribuição da LTA deixaram clara esta influência, em que $L$. intermedia e $L$. migonei foram as espécies encontradas em altitudes de 0 a 700m do nível do mar.

Comparando-se o conjunto das cinco espécies com a média dos coeficientes padronizados de incidência entre os municípios, não se assinalou diferença significativa $(p=0,475)$, porquanto, a análise comparativa entre os municípios em que foi detectada a presença de pelo menos uma das cinco espécies de flebotomíneos e àqueles em que a pesquisa entomológica foi negativa apresentou diferença significativa $(p=0,026)$ em relação à média dos coeficientes padronizados de incidência. Em ambos casos parecem confirmar que a doença existe onde tem vetor e que a outra explicação para a ausência do flebotomíneo nas localidades com casos humanos poderia estar relacionada às capturas executadas tardiamente em relação ao momento da transmissão, o que coincidiria com período de baixa densidade das espécies. Deve-se considerar, também, que o sucesso de uma captura entomológica está associado à agilidade na identificação de focos da doença pelos serviços de vigilância, dos recursos humanos disponíveis, para a realização da captura na época mais propícia. Por outro lado, o atraso na identificação destes focos está associado ao longo período de incubação da doença, ao retardamento do paciente a procura pela assistência médica e o tempo de obtenção do diagnóstico laboratorial, como já apontado anteriormente por Gomes \& Camargo-Neves ${ }^{14}$.

Outra diferença significativa foi observada quando comparadas às médias dos coeficientes padronizados de incidência de LTA entre os municípios em que foram realizadas as pesquisas de flebotomíneos e àqueles em que a pesquisa não foi realizada $(p<0,001)$ indicando que o baixo registro de casos deve estar relacionado à baixa densidade vetorial.

$\mathrm{Na}$ análise realizada entre as mesmas espécies observou-se duas diferenças que foram significativas ( $p<0,1)$, indicando que $L$. pessoai foi a espécie que esteve associada a um nível de incidência de LTA significativamente menos elevado que $L$. migonei $(p=0,073)$ e $L$. fischeri $(p=0,055)$. Uma possível diferença a ser considerada seria que ecologicamente L. pessoai e L. migonei ocupam nichos diferentes, por vezes com ocorrência alopátrica, ou seja, a primeira ocorreria apenas nas matas preservadas, enquanto L. migonei se distribuiria por esses e outros habitats. Daí, ser possível admitir papéis vetoriais em ambientes diferenciados, principalmente quando esse aspecto parece ser ainda influenciado por fatores topográficos que sabidamente delimitam a presença e abundância dos flebotomíneos ${ }^{15}$. Em área simpátrica de serra, no Estado do Ceará, L. migonei foi encontrada naturalmente infectada com L. braziliensis e junto com $L$. whitmani foram incriminadas como transmissoras da $\mathrm{LTA}^{2}{ }^{3}$.

A cobertura florestal foi no passado o ambiente mais infeccioso para humanos. Ainda hoje, ele representa um importante indicador de risco, pois detém a enzootia selvática. A evidência dessa afirmação está na análise comparativa entre os tipos de cobertura florestal e a média dos coeficientes padronizados de incidência, demonstrando que o tipo de vegetação influiu significativamente no coeficiente de incidência $(p<0,001)$. Se ainda for considerada a predominância do ambiente alterado, incluindo aqueles, onde a transmissão da LTA ocorreu, não existirá dúvida que o 
aspecto paisagístico primitivo sofreu muita alteração e com ele as taxas de incidência se alteraram. Daí a razão dos focos naturais remanescentes aproximarem-se do ambiente domiciliar. Corrobora com essa situação o encontro de $75,6 \%$ dos municípios com transmissão, apresentarem vegetação natural bastante modificada e indivíduos arbóreos menores como cerrado e capoeira. Este aspecto desfavorece o encontro de $L$. whitmani, mas L. migoneitem sido predominante junto com $L$. intermedia.

Como os focos selváticos da doença vêm sendo cada vez mais raros, L. whitmani pode ter perdido sua importância epidemiológica nas áreas modificadas do Estado de São Paulo, assim como à L. pessoai ${ }^{13}$. Contudo, Condino e cols ${ }^{5}$, investigando a transmissão da LTA no município de Teodoro Sampaio, demonstraram a importância desta espécie juntamente com L. intermedia, reforçando seu papel vetorial em áreas de mata remanescentes no extremo oeste do estado.

Infelizmente, o caráter qualitativo das informações utilizadas dificultou uma análise com direcionamento mais específico para as espécies flebotomíneas estudadas, principalmente, por não se dispor de dados de densidade para elucidar melhor a expansão territorial dos casos humanos.

As áreas de transmissão da doença, também, foram analisadas segundo modelo de regressão linear múltipla, desta forma foi possível definir aquelas com maior incidência de LTA, ou seja, a região compreendida pelo Planalto Atlântico $(p=0,005)$ e as áreas com cobertura vegetal natural do Tipo $V$ - mata $(p=0,000)$, destacando-se a participação de $L$. migonei $(\mathrm{p}=0,029)$. Portanto, a presença de $L$. migonei foi assinalada nas áreas onde foram registrados os valores mais altos das médias dos coeficientes padronizados de incidência, ou seja, nos ambientes alterados ou do tipo mata residual, ambas favoráveis à presença dessa espécie. Com isto, inclina-se a suspeitar que $L$. migonei desempenhe, também, papel vetorial na LTA, porém naquelas áreas relacionadas com médias de temperaturas mais elevadas $(p=0,001)$. Para a área peridoméstica de Baturité, CE, L. whitmani e L. migonei foram as espécies predominantes ${ }^{1}$, mas a primeira espécie foi mais capturada com isca humana, enquanto $L$. migonei com isca animal, ao contrário do que foi detectado no Estado de São Paulo, onde se observou um hábito eclético, mas com avidez pelo sangue humano ${ }^{10}$.

Diante dessas evidências, os autores admitem o envolvimento de mais de uma espécie na transmissão da L. braziliensis. Os resultados deste trabalho mostram, mais claramente, que $L$. intermedia foi a espécie mais abundante, mas, talvez, dado ao seu alto grau de endofilia, apresente menor infecciosidade no ambiente domiciliar do que L. migonei, se considerar que, inicialmente, não há fonte de infecção primária neste ambiente. Apesar de ambas espécies estarem presentes nas áreas de maiores incidências da doença, L. migonei esteve associada com os maiores coeficientes de incidência. Isto pode ser explicado pelo seu comportamento tanto zoofílico quanto antropofílico, consequentemente haveria maior chance de se infectar em reservatórios primários e em animais domésticos como o cão ${ }^{78}$. Mesmo assim, fazem-se necessários novos estudos sobre a infecção natural e hábito alimentar destas espécies para a elucidação dessa hipótese.

Face ao exposto, o presente estudo retoma à discussão sobre o status de $L$. migonei entre as espécies flebotomíneas que veiculam a LTA no Estado de São Paulo, sobretudo resgatando o papel epidemiológico já evidenciado em investigações mais antigas ${ }^{9}$.

Finalmente, parece não haver dúvida que haja envolvimento de mais de uma espécie vetora na transmissão da leishmaniose tegumentar no Estado de São Paulo. Este estudo retomou a discussão sobre o papel de cinco espécies de flebotomíneos, todavia destacando para o ambiente antrópico o possível papel da L. intermedia e L. migonei. Além disso, os autores levantam questões fundamentais ao aprimoramento dos programas de controle da LTA em nosso estado.

\section{REFERÊNCIAS BIBLIOGRÁFICAS}

1. Azevedo ACR, Rangel EF. A study of sandfly species (Diptera: Psychodidae: Phlebotominae) in focus of cutaneous Leishmaniasis in the municipality of Baturité, Ceará, Brazil. Memórias do Instituto Oswaldo Cruz 86: 405-410, 1991.

2. Azevedo ACR, Rangel EF, Costa EM, David J, Vasconcelos AW, Lopes UG. Natural infection of Lutzomyia (Nyssomyia) whitmani (Antunes \& Coutinho, 1939) by Leishmania of the Braziliensis complex in Baturité, Ceará State, Northeast Brazil. Memórias do Instituto Oswaldo Cruz 85: 251, 1990.

3. Azevedo ACR, Rangel EF, Queiroz RG. Lutzomyia migonei (França, 1920) naturally infected with peripylarian flagellates in Baturité, a focus of leishmaniasis in Ceará State, Brazil. Memórias do Instituto Oswaldo Cruz 85: 479, 1990.

4. Camargo-Neves VLF. Características da Transmissão da Leishmaniose Tegumentar Americana no Estado de São Paulo, Brasil. Dissertação de Mestrado, Faculdade de Saúde Pública da Universidade de São Paulo, São Paulo, SP, 1999.
5. Condino MLF, Sampaio SMP, Henriques LF, Galati EAB, Wanderley DMV, Corrêa FMA. Leishmaniose tegumentar americana: Flebotomíneos do Estado de São Paulo, Brasil. Revista da Sociedade Brasileira de Medicina Tropical 31 : 355-360, 1998.

6. Daniel WW. Bioestatistics. A Foundation for Analysis in the Health Sciences. 6 $6^{\text {a }}$ edition. John Wiley \& Sons, New York, EUA, 1995.

7. Falqueto A. Especificidade alimentar de flebotomíneos em duas áreas endêmicas de Leishmaniose Tegumentar no Estado de Espírito Santo. Tese de Doutorado, Instituto Oswaldo Cruz da Fundação Oswaldo Cruz, Rio de Janeiro, RJ, 1995.

8. Falqueto A, Coura JR, Barros GC, Grimaldi JR, Sessa PA, Carias VRD, Jesus AC, Alencar JTA. Participação do cão no ciclo de transmissão da Leishmaniose tegumentar no município de Viana, Estado do Espírito Santo, Brasil. Memórias do Instituto Oswaldo Cruz 81: 155-163, 1986. 
9. Forattini OP. Subfamília Phlebotominae - Biologia. In: Entomologia Médica. Editora da Universidade de São Paulo, São Paulo, 1973.

10. Forattini OP. Algumas observações sobre a biologia de flebótomos em região da Bacia do Paraná (Brasil). Arquivos da Faculdade de Higiene São Paulo 7: 157-167, 1954.

11. Forattini OP, Rabello EX, Serra OP, Cotrim MD, Galati EAB, Barata JMS. Observações sobre a transmissão da leishmaniose tegumentar no Estado de São Paulo, Brasil. Revista de Saúde Pública 10: 31-43, 1976.

12. Frees EW. Data analysis using Regression models. In: The business perspective. Englewood Cliffs, New Jersey, USA, 714 pp, 1996.

13. Gomes AC. Perfil epidemiológico da Leishmaniose Tegumentar Americana no Brasil. Revista Brasileira de Dermatologia 67: 55- 60, 1992.

14. Gomes AC, Camargo-Neves VLF. Estratégias e perspectivas de controle da Leishmaniose Tegumentar Americana no Estado de São Paulo. Revista da Sociedade Brasileira de Medicina Tropical 31: 553-558, 1998.

15. Gomes AC, Galati EAB, Casanova C, Domingos MF, Marques GRAM, Camargo-Neves VLF. Analysis of the geographical distribution of Leishmaniasis Vectors in State of São Paulo. Boletín de la Dirección de Malariología y Saneamiento Ambiental 35: 143-146, 1995.

16. Instituto Brasileiro de Geografia e Estatística. Instituto Geográfico e Cartográfico de Economia e Planejamento do Estado de São Paulo. Secretaria de Economia e Planejamento do Estado de São Paulo. Mapa das divisões geomorfológicas do Estado de São Paulo. São Paulo, SP, 1982.

17. Kronka FJN, Matsukuma CK, Nalon MA, Cali IH, Rossi M, Mattos IFA, Shin-lke MS, Pontinhas AS. Inventário Florestal do Estado de São Paulo, p.1-199, 1993.

18. Pessôa SB, Barretto M.P. Leishmaniose Tegumentar Americana. Ministério da Educação e Saúde, Serviço de Documentação, Imprensa Nacional, Rio de Janeiro, 1948.

19. Secretaria de Estado da Saúde. Manual de Vigilância Epidemiológica - Leishmaniose Tegumentar Americana, Normas e Instruções. Centro de Vigilância Epidemiológica, São Paulo, 1995.

20. Young DG, Duncan MA. Guide to the Identification and Geographic Distribution of Lutzomyia Sand Flies in Mexico, the West Indies, Central and South America (Diptera: Psychodidae). Associated Publishers, Gainsville, Florida, USA, 1994. 